\title{
Bounded Linear Stability Margin Analysis of Nonlinear Hybrid Adaptive Control
}

\author{
Nhan T. Nguyen, Jovan D. Boskovic
}

\begin{abstract}
This paper presents a bounded linear stability analysis for a hybrid adaptive control that blends both direct and indirect adaptive control. Stability and convergence of nonlinear adaptive control are analyzed using an approximate linear equivalent system. A stability margin analysis shows that a large adaptive gain can lead to a reduced phase margin. This method can enable metrics-driven adaptive control whereby the adaptive gain is adjusted to meet stability margin requirements.
\end{abstract}

\section{INTRODUCTION}

Adaptive control is nonlinear and stability of adaptive control cannot be analyzed by the traditional phase and gain margins. These margins are used for linear control laws to provide robustness in the presence of system uncertainties. The lack of stability metrics for adaptive control is a major challenge to certifying adaptive control for safety-critical systems. Metrics-driven adaptive control introduces a notion that adaptation should be driven by some stability metrics to achieve robustness [1]. A bounded linear stability analysis method is introduced for analyzing adaptive control in terms of the linear stability concept by establishing an approximate linear equivalent system as a function of persistent excitation. This linear equivalent system is only used for analysis and not for actual adaptation, and can provide estimates of relative stability of nonlinear adaptive control for a given adaptive gain. By adjusting the adaptive gain during the adaptation to meet certain stability margin requirements, the adaptive law is thus made to be metrics-driven. The bounded linear stability analysis is studied in a framework of a hybrid adaptive control which blends both direct and indirect adaptive control to improve tracking performance [2], as shown in Fig. 1.

In recent years, direct model-reference adaptive control (MRAC) using neural networks has been a topic of great research interests [3], [4], [5]. Indirect adaptive control achieves adaptation by means of system identification of plant parameters or uncertainties based on certaintyequivalence control schemes [6], [7]. In this study, a recursive least-squares (RLS) indirect adaptive law is used as a parameter estimation technique to reduce the modeling error, while a direct MRAC law achieves a reduction in

Nhan T. Nguyen is with the Intelligent Systems Division at NASA Ames Research Center, Moffett Field, CA 94035 USA (e-mail: Nhan.T.Nguyen@nasa.gov).

Jovan D. Boskovic is with Scientific Systems Company, Inc., Woburn, MA 01801 USA (e-mail: Jovan.Boskovic@ssci.com). the tracking error. The study shows that the hybrid adaptive control potentially can offer better tracking performance and can prevent problems with high-gain control using direct MRAC alone.

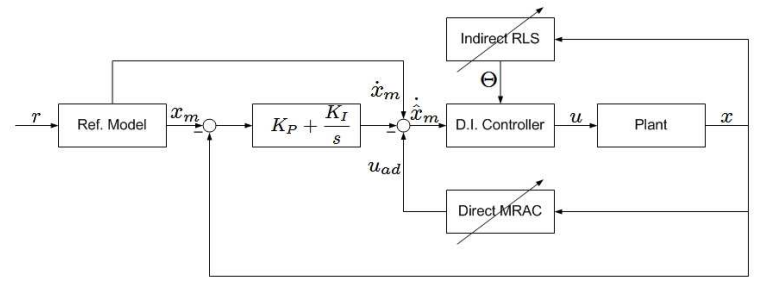

Fig. 1 - Hybrid Adaptive Control Architecture

\section{HyBRID ADAPTIVE CONTROL}

Given a plant model as

$$
\dot{x}=A_{p} x+B_{p} u
$$

where $x \in \mathbb{R}^{n}$ is a state vector, $u \in \mathbb{R}^{n}$ is a control vector, and $A_{p}, B_{p} \in \mathbb{R}^{n \times n}$ are unknown.

The objective is to produce a controller that enables the plant to follow a reference model described by

$$
\dot{x}_{m}=A_{m} x_{m}+B_{m} r
$$

where $A_{m} \in \mathbb{R}^{n \times n}$ is Hurwitz and given, $B_{m} \in \mathbb{R}^{n \times n}$ is also given, and $r \in \mathbb{R}^{n} \in \mathscr{L}_{\infty}$ is a bounded command vector with $\dot{r} \in \mathbb{R}^{n} \in \mathscr{L}_{\infty}$ also bounded.

Defining an estimator model

$$
\dot{\hat{x}}=A x+B u+\Theta^{\top} \Phi+u_{a d}
$$

where $A, B \in \mathbb{R}^{n \times n}$ are known, $\Theta^{\top}=\left[\begin{array}{cc}\Delta \hat{A} & \Delta \hat{B}\end{array}\right] \in \mathbb{R}^{n \times 2 n}$, $\Phi=\left[\begin{array}{ll}x^{\top} & u^{\top}\end{array}\right]^{\top} \in \mathbb{R}^{2 n}$, and $u_{a d} \in \mathbb{R}^{n}$ is a direct adaptive signal.

Defining the tracking error as $\tilde{x}=x_{m}-x$, the goal is to determine a controller that results in $\lim _{t \rightarrow \infty}\|\tilde{x}\|=0$. A dynamic inversion controller is designed from Eq. (3) to give the tracking error a second-order response with a proportional-integral feedback control as

$$
u=\hat{B}_{p}^{-1}\left(\dot{x}_{m}-\hat{A}_{p} x+K_{p} \tilde{x}+K_{i} \int_{0}^{t} \tilde{x} d \tau-u_{a d}\right)
$$

where $\hat{A}_{p}=A+\Delta \hat{A}$ and $\hat{B}_{p}=B+\Delta \hat{B}$ are estimates of $A_{p}$ and $B_{p}, K_{p}=\operatorname{diag}\left(k_{p, 1}, \ldots, k_{p, n}\right)>0$, and $K_{i}=$ $\operatorname{diag}\left(k_{i, 1}, \ldots, k_{i, n}\right)>0$. 
The tracking error dynamics are expressed as

$$
\dot{\tilde{x}}=-K_{p} \tilde{x}-K_{i} \int_{0}^{t} \tilde{x} d \tau+\Theta^{\top} \Phi+u_{a d}-\left(A_{p}-A\right) x-\left(B_{p}-B\right) u
$$$$
\text { Let } e=\left[\begin{array}{ll}
\int_{0}^{t} \tilde{x} d \tau & \tilde{x}
\end{array}\right]^{\top} \in \mathbb{R}^{2 n} \text {, then }
$$

$$
\dot{e}=A_{c} e+b\left(\Theta^{\top} \Phi+u_{a d}-\varepsilon\right)
$$

where $\varepsilon=\dot{x}-A x-B u$ is an estimation error which is assumed to be measurable and

$$
A_{c}=\left[\begin{array}{cc}
0 & I \\
-K_{i} & -K_{p}
\end{array}\right], \quad b=\left[\begin{array}{l}
0 \\
I
\end{array}\right]
$$

The direct adaptive signal is parameterized by a linear-inparameter matched uncertainty as

$$
u_{a d}=W^{\top} \beta(x)
$$

where $W \in \mathbb{R}^{m \times n}$ is a weight matrix and $\beta \in \mathbb{R}^{m}$ is a basis vector with Lipschitz properties

$$
\left\|\beta(x)-\beta\left(x_{0}\right)\right\| \leq C\left\|x-x_{0}\right\|
$$

for some constant $C>0$, which implies a bounded derivative

$$
\left\|\frac{\partial \beta(x)}{\partial x}\right\| \leq L
$$

for some constant $L>0$.

The adaptive law is given by

$$
\dot{W}=-\Gamma \beta e^{\top} P b
$$

where $\Gamma>0 \in \mathbb{R}$ is an adaptive gain and $P>0 \in \mathbb{R}^{2 n \times 2 n}$ solves the Lyapunov equation

$$
P A_{c}+A_{c}^{\top} P=-Q
$$

where $Q>0$ is a symmetric positive-definite matrix.

$\Delta \hat{A}$ and $\Delta \hat{B}$ are estimated by an indirect adaptive law based on the recursive least-squares (RLS) method

$$
\begin{gathered}
\dot{\Theta}=-\frac{1}{m^{2}} R \Phi\left(\Phi^{\top} \Theta-\varepsilon^{\top}\right) \\
\dot{R}=-\frac{1}{m^{2}} R \Phi \Phi^{\top} R
\end{gathered}
$$

where $m^{2}=1+\Phi^{\top} R \Phi \in \mathbb{R}$ is a normalization factor, $R>$ $0 \in \mathbb{R}^{2 n \times 2 n}$ is a covariance matrix.

The proof of the RLS indirect adaptive law is as follows:

Proof: Consider the following cost functional to be minimized

$$
J(\Theta)=\frac{1}{2} \int_{0}^{t} \frac{1}{m^{2}}\left\|\Theta^{\top} \Phi-\varepsilon\right\|^{2} d \tau
$$

The necessary condition is obtained as

$$
\nabla J_{\Theta}^{\top}=0 \Rightarrow \int_{0}^{t} \frac{1}{m^{2}} \Phi \Phi^{\top} \Theta d \tau=\int_{0}^{t} \frac{1}{m^{2}} \Phi \varepsilon^{\top} d \tau
$$

By letting

$$
R^{-1}=\int_{0}^{t} \frac{1}{m^{2}} \Phi \Phi^{\top} d \tau
$$

then it can be shown that

$$
R^{-1} \dot{\Theta}+\frac{1}{m^{2}} \Phi \Phi^{\top} \Theta=\frac{1}{m^{2}} \Phi \varepsilon^{\top}
$$

which results in Eq. (13). Differentiation of the identity $R^{-1} R=I$ also yields Eq. (14).

Proposition 1: The hybrid adaptive law can be shown to be stable and result in bounded signals.

Proof: Let $\Theta^{*}, W^{*}$ be constant ideal weights, and $\tilde{\Theta}=$ $\Theta-\Theta^{*}, \tilde{W}=W-W^{*}$ be weight variations, then $\dot{\tilde{\Theta}}=\dot{\Theta}$ and $\dot{\tilde{W}}=\dot{W}$. Consider the following Lyapunov candidate function

$$
V=e^{\top} P e+\operatorname{trace}\left(\tilde{W}^{\top} \Gamma^{-1} \tilde{W}+\tilde{\Theta}^{\top} R^{-1} \tilde{\Theta}\right)
$$

$\dot{V}$ is evaluated as

$$
\begin{array}{r}
\dot{V}=e\left(A_{c}^{\top} P+P A_{c}\right) e+2 e^{\top} P b\left(\Theta^{\top} \Phi+W^{\top} \beta-\varepsilon\right) \\
+\operatorname{trace}\left[-2 \tilde{W}^{\top} \beta e^{\top} P b-\frac{2}{m^{2}} \tilde{\Theta}^{\top} \Phi\left(\Phi^{\top} \Theta-\varepsilon^{\top}\right)\right. \\
\left.+\tilde{\Theta}^{\top} \frac{d}{d t}\left(R^{-1}\right) \tilde{\Theta}\right]
\end{array}
$$

Since $R^{-1} R=I$, then

$$
\begin{aligned}
& \frac{d}{d t}\left(R^{-1}\right) R+R^{-1} \dot{R}=\frac{d}{d t}\left(R^{-1}\right) R-\frac{1}{m^{2}} \Phi \Phi^{\top} R=0 \\
& \text { So } \begin{array}{l}
\frac{d}{d t}\left(R^{-1}\right)=\frac{1}{m^{2}} \Phi \Phi^{\top}
\end{array}
\end{aligned}
$$

Using the trace property trace $(A B)=B A$, one then obtains

$$
\begin{aligned}
\dot{V} \leq-e^{\top} Q e+2 e^{\top} P b\left(\tilde{\Theta}^{\top} \Phi+\Delta_{2}\right) & \\
- & \frac{2}{m^{2}}\left(\Phi^{\top} \tilde{\Theta}-\Delta_{1}^{\top}\right) \tilde{\Theta}^{\top} \Phi+\frac{1}{m^{2}} \Phi^{\top} \tilde{\Theta} \tilde{\Theta}^{\top} \Phi
\end{aligned}
$$

where $\Delta_{1}=\sup _{x, u}\left|\varepsilon-\Theta^{* \top} \Phi\right|$ and $\Delta_{2}=\sup _{x, u}\left|W^{* \top} \beta-\Delta_{1}\right|$ are approximation errors.

$\dot{V}$ is bounded by

$$
\begin{gathered}
\dot{V} \leq-\lambda_{\min }(Q)\|e\|^{2}+2 \lambda_{\max }(P)\|e\|\left(\left\|\tilde{\Theta}^{\top} \Phi\right\|+\left\|\Delta_{2}\right\|\right) \\
-\frac{1}{m^{2}}\left\|\tilde{\Theta}^{\top} \Phi\right\|^{2}+\frac{2}{m^{2}}\left\|\Delta_{1}\right\|\left\|\tilde{\Theta}^{\top} \Phi\right\| \\
=-\|e\|\left[\lambda_{\min }(Q)\|e\|-2 \lambda_{\max }(P)\left\|\Delta_{2}\right\|\right] \\
-\left\|\tilde{\Theta}^{\top} \Phi\right\|\left[\frac{1}{m^{2}}\left\|\tilde{\Theta}^{\top} \Phi\right\|-2 \lambda_{\max }(P)\|e\|-\frac{2}{m^{2}}\left\|\Delta_{1}\right\|\right]
\end{gathered}
$$

Defining a compact set $\mathscr{V}$ as

$$
\begin{array}{r}
\mathscr{V}=\left\{e \in \mathbb{R}^{n}, \tilde{\Theta}^{\top} \Phi \in \mathbb{R}^{n}:\|e\| \geq r_{1}=\frac{2 \lambda_{\max }(P)\left\|\Delta_{2}\right\|}{\lambda_{\min }(Q)},\right. \\
\left.\left\|\tilde{\Theta}^{\top} \Phi\right\| \geq r_{2}=2 r_{1} m^{2} \lambda_{\text {max }}(P)+2\left\|\Delta_{1}\right\|\right\}
\end{array}
$$

and a complementary compact set $\mathscr{S}$ which contains $e=0$ and $\tilde{\Theta}=0$, then $V$ increases in $\mathscr{S}$ but all trajectories of $e$ and $\tilde{\Theta}^{\top} \Phi$ will stay inside of $\mathscr{S}$. It follows by LaSalle's extensions of the Lyapunov method that $e$ and $\tilde{\Theta}$ are bounded, and so are $x$ and $u$. 


\section{Bounded Linear Stability ANALYSiS}

Stability of nonlinear adaptive control is usually analyzed by the Lyapunov method. The traditional linear stability margin concept may be extended to nonlinear adaptive control if it could be represented by some linear approximations. To obtain an equivalent LTI system, the adaptive law can be linearized at a certain point in time when the weights are at a steady state, usually long after initial transients have settled down. However, transient responses during adaptation can be important and the adaptive law should be designed in a way that would prevent large initial transients which can compromise system robustness. The bounded linear stability analysis seeks a piecewise linear equivalent approximation of nonlinear adaptive control in terms of a persistent excitation (PE) over a short, moving time window during which the LTI concept of stability margins could be analyzed to provide a method for adjusting the adaptive gain for the next time window. The linear equivalent approximation is not a replacement of an adaptive law but rather is used in conjunction with the adaptive law for the stability analysis purpose.

Theorem 1: The hybrid adaptive law and the tracking error dynamics can be approximated by a piecewise linear representation as

$$
\begin{aligned}
\frac{d}{d t}\left[\begin{array}{c}
e \\
z_{1} \\
z_{2}
\end{array}\right] \leq\left[\begin{array}{ccc}
A_{c} & b & b \\
-\Gamma \beta_{0}^{2} b^{\top} P & 0 & 0 \\
0 & 0 & -a
\end{array}\right] & {\left[\begin{array}{c}
e \\
z_{1} \\
z_{2}
\end{array}\right] } \\
& +\left[\begin{array}{c}
b \Delta_{2} \\
\varepsilon_{1} \\
\varepsilon_{2}
\end{array}\right]
\end{aligned}
$$

over a semi-open time interval $t \in\left(t_{0}-T, t_{0}\right]$, where $z_{1}, z_{2} \in$ $\mathbb{R}^{n}, a=\frac{R_{0} \Phi_{0}^{2}}{1+R_{0} \Phi_{0}^{2}}>0, R_{0}=\lambda_{\text {min }}(R)$, and $\beta_{0}^{2}, \Phi_{0}^{2} \in \mathbb{R}$ are persistent excitation values defined as

$$
\begin{aligned}
& \beta_{0}^{2}=\frac{1}{T} \int_{t_{0}-T}^{t_{0}} \beta^{\top} \beta d t \\
& \Phi_{0}^{2}=\frac{1}{T} \int_{t_{0}-T}^{t_{0}} \Phi^{\top} \Phi d t
\end{aligned}
$$

Let $z_{1}=\tilde{W}^{\top} \beta \in \mathbb{R}^{n}$ and $z_{2}=\tilde{\Theta}^{\top} \Phi \in \mathbb{R}^{n}$. Then

$$
\begin{gathered}
\dot{z}_{1}=-b^{\top} P e \beta^{\top} \Gamma \beta+\tilde{W}^{\top} \dot{\beta} \\
\dot{z}_{2}=-\frac{1}{m^{2}} z_{2} \Phi^{\top} R \Phi+\frac{1}{m^{2}}\left(\varepsilon-\Theta^{* \top} \Phi\right) \Phi^{\top} R \Phi+\tilde{\Theta}^{\top} \dot{\Phi}
\end{gathered}
$$

Since $\beta$ satisfies the Lipschitz condition and $\dot{x}$ is bounded because $x$ and $u$ are bounded, then $\dot{\beta}=\frac{\partial \beta}{\partial x} \dot{x}$ is therefore bounded. Also, $\dot{\Phi}$ is bounded since $\dot{\Phi}=\left[\begin{array}{cc}\dot{x}^{\top} & \dot{u}^{\top}\end{array}\right]^{\top}$ and $\dot{u}$ can be shown to be bounded by differentiating Eq. (4) as

$$
\begin{aligned}
\dot{u} & =\hat{B}_{p}^{-1}\left[A_{m} \dot{x}_{m}+B_{m} \dot{r}-\hat{A}_{p} \dot{x}-b^{\top} A_{c} \dot{e}\right. \\
& \left.+\frac{1}{m^{2}}\left(\Theta^{\top} \Phi-\varepsilon\right) \Phi^{\top} R \Phi+b^{\top} P e \beta^{\top} \Gamma \beta-W^{\top} \dot{\beta}\right]
\end{aligned}
$$

Let $\varepsilon_{1}=\sup _{x, u, t}\left|\tilde{W}^{\top} \dot{\beta}\right|$ and $\varepsilon_{2}=\sup _{x, u, t}\left|\frac{\Phi^{\top} R \Phi}{m^{2}} \Delta_{1}+\tilde{\Theta}^{\top} \dot{\Phi}\right|$ for $t \in\left(t_{0}-T, t_{0}\right]$. These error terms come from the actual adaptive laws (11) and (13) and thus act as bounded disturbances. Upon integration, one gets

$$
\begin{aligned}
& z_{1}\left(t_{0}\right)-z_{1}\left(t_{0}-T\right) \leq-\int_{t_{0}-T}^{t_{0}} b^{\top} P e \beta^{\top} \Gamma \beta d t+\varepsilon_{1} T \\
& z_{2}\left(t_{0}\right)-z_{2}\left(t_{0}-T\right) \leq-\int_{t_{0}-T}^{t_{0}} \frac{1}{m^{2}} z_{2} \Phi^{\top} R \Phi d t+\varepsilon_{2} T
\end{aligned}
$$

The mean value theorem for integration states that

$$
\int_{a}^{b} F(t) G(t) d t=F(c) \int_{a}^{b} G(t) d t
$$

where $c \in[a, b]$ and $\mathrm{g}(t) \geq 0$. If $G=1$, then the special case of the mean value theorem for integration is obtained as

$$
\int_{a}^{b} F(t) d t=F(c)(b-a)
$$

Applying the mean value theorem for integration then yields

$$
\begin{aligned}
& z_{1}\left(t_{0}\right)-z_{1}\left(t_{0}-T\right) \leq-\Gamma b^{\top} P e\left(t_{1}\right) \int_{t_{0}-T}^{t_{0}} \beta^{\top} \beta d t+\varepsilon_{1} T \\
& z_{2}\left(t_{0}\right)-z_{2}\left(t_{0}-T\right) \leq-z_{2}\left(t_{1}\right) \int_{t_{0}-T}^{t_{0}} \frac{1}{m^{2}} \Phi^{\top} R \Phi d t+\varepsilon_{2} T
\end{aligned}
$$

where $t_{1} \in\left(t_{0}-T, t_{0}\right]$.

But $R_{0} \Phi^{\top} \Phi \leq \Phi^{\top} R \Phi$, hence

$$
\begin{array}{r}
z_{2}\left(t_{0}\right)-z_{2}\left(t_{0}-T\right) \leq-\frac{R_{0}}{1+R_{0} \Phi\left(t_{1}\right)^{\top} \Phi\left(t_{1}\right)} z_{2}\left(t_{1}\right) \times \\
\times \int_{t_{0}-T}^{t_{0}} \Phi^{\top} \Phi d t+\varepsilon_{2} T
\end{array}
$$

Applying the mean value theorem for integration once more gives

$$
\int_{t_{0}-T}^{t_{0}} \Phi^{\top} \Phi d t=\Phi\left(t_{2}\right)^{\top} \Phi\left(t_{2}\right) T
$$

If $T$ is sufficiently small, then $t_{1} \approx t_{2} \approx t \in\left(t_{0}-T, t_{0}\right]$ so that

$$
\Phi\left(t_{1}\right)^{\top} \Phi\left(t_{1}\right) \approx \Phi\left(t_{2}\right)^{\top} \Phi\left(t_{2}\right)=\frac{1}{T} \int_{t_{0}-T}^{t_{0}} \Phi^{\top} \Phi d t=\Phi_{0}^{2}
$$

and

$$
\begin{gathered}
\dot{z}_{1} \approx \frac{z_{1}\left(t_{0}\right)-z_{1}\left(t_{0}-T\right)}{T} \leq-\Gamma_{0} \beta_{0}^{2} b^{\top} P e+\varepsilon_{1} \\
\dot{z}_{2} \approx \frac{z_{2}\left(t_{0}\right)-z_{2}\left(t_{0}-T\right)}{T} \leq-a z_{2}+\varepsilon_{2}
\end{gathered}
$$

The tracking error dynamics can also be written as

$$
\dot{e} \leq A_{c} e+b\left(z_{1}+z_{2}+\Delta_{2}\right)
$$

Remark 1: The piecewise linear approximation of the nonlinear adaptive laws and the tracking error dynamics over a moving time window enables the adaptive control to be analyzed in the context of an equivalent LTI system from which system robustness can be assessed via the 
linear stability margin concept during that time window. The window width $T$ can be adjusted to sufficiently capture initial transients for analyzing system robustness.

Remark 2: The persistent excitation values $\beta_{0}^{2}$ and $\Phi_{0}^{2}$ may be a more suitable choice than the standard persistent excitation definition which would be $\frac{1}{T} \int_{t_{0}-T}^{t_{0}} \beta \beta^{\top} d t$ and $\frac{1}{T} \int_{t_{0}-T}^{t_{0}} \Phi \Phi^{\top} d t$, respectively. The persistent excitation matrices are singular and so are not invertible. On the other hand, $\beta_{0}^{2}$ and $\Phi_{0}^{2}$ are zero only if $\beta=0$ and $\Phi=0$. It can be shown that the tracking error depends on $\beta_{0}^{2}$ and the approximation error of the direct adaptive law, while $\Phi_{0}^{2}$ affects the parameter convergence of the RLS indirect adaptive law.

Proof: Eliminating $z_{1}$ and $z_{2}$ in the linearly approximate tracking error dynamics results in

$$
\left(s^{2}-A_{c} s+\Gamma \beta_{0}^{2} b b^{\top} P\right) e \leq b\left(-\frac{R_{0} \Phi_{0}^{2}}{1+R_{0} \Phi_{0}^{2}} z_{2}+\varepsilon_{1}+\varepsilon_{2}+\Delta_{2}\right)
$$

For $R_{0} \Phi_{0}^{2} \gg 1, a \approx 1$, the solution of $z_{2}$ is

$$
z_{2}\left(t_{0}\right) \leq\left[z_{2}\left(t_{0}-T\right)-\varepsilon_{2}\right] e^{-T}+\varepsilon_{2}
$$

so in the limit $z_{2}$ converges to

$$
\lim _{t_{0} \rightarrow \infty} \sup \left|z_{2}\right|=\varepsilon_{2}
$$

Therefore, the convergence of the tracking error can be found by

$$
\lim _{t_{0} \rightarrow \infty} \sup |e|=\frac{b\left(\varepsilon_{1}+\Delta_{2}\right)}{\Gamma \beta_{0}^{2} \lambda_{\text {min }}\left(b b^{\top} P\right)}
$$

One should note that while increasing $\Gamma \beta_{0}^{2}$ can help reduce the tracking error, the system robustness may be compromised when it is examined in the context of the LTI stability margins.

To analyze the linear stability of the approximate tracking error and the hybrid adaptive law, the characteristic equation of closed-loop system is evaluated by the Schur complement formula as

$$
\operatorname{det}(s I-\bar{A})=\operatorname{det}(s I+a I) s \operatorname{det}\left(s I-A_{c}+\frac{\Gamma \beta_{0}^{2} b b^{\top} P}{s}\right)
$$

where $\bar{A}$ is the state transition matrix in Eq. (26).

Since $K_{p}$ and $K_{i}$ are diagonal and represent individual loop gains for the tracking error, the determinant can be evaluated as

$$
\begin{aligned}
& \operatorname{det}(s I-\bar{A})=(s+a)^{n} \times \\
& \quad \times \prod_{i=1}^{n}\left(s^{3}+k_{p, i} s^{2}+k_{i, i} s+\Gamma \beta_{0}^{2} p_{22, i} s+\Gamma \beta_{0}^{2} p_{12, i}\right)
\end{aligned}
$$

where $p_{12, i}=q k_{i, i}^{-1}$ and $p_{22, i}=q k_{p, i}^{-1}\left(1+k_{i, i}^{-1}\right), i=1, \ldots, n$, are diagonal elements of partitioned matrices $P_{12}$ and $P_{22}$ of $P$, which solves Eq. (12) with $Q=2 q I$, where $q>0$ is a constant.

The linear equivalent effect of the RLS indirect adaptive law is to add a pole at $s=-a$, but it does not interact with the tracking error dynamics. On the other hand, the direct MRAC interacts intimately with the tracking error which can affect robustness of the direct adaptive law. For each loop, the characteristic equation is

$$
(s+a)\left(s^{3}+k_{p} s^{2}+k_{i} s+\Gamma \beta_{0}^{2} p_{22} s+\Gamma \beta_{0}^{2} p_{12}\right)=0
$$

For brevity, the subscript $i$ is dropped. By factorization with residue, the characteristic equation can be written as

$$
\begin{array}{r}
(s+a)\left\{( s + \Gamma \beta _ { 0 } ^ { 2 } \alpha ) \left[s^{2}+\left(k_{p}-\Gamma \beta_{0}^{2} \alpha\right) s+k_{i}+\Gamma \beta_{0}^{2} p_{22}\right.\right. \\
\left.\left.-\Gamma \beta_{0}^{2} \alpha\left(k_{p}-\Gamma \beta_{0}^{2} \alpha\right)\right]+r\right\}=0
\end{array}
$$

where $a$ and the residue $r$ are defined as

$$
\begin{gathered}
\alpha=\left(k_{i}+\Gamma \beta_{0}^{2} p_{22}\right)^{-1} p_{12} \\
r=\Gamma \beta_{0}^{2} p_{12}-\Gamma \beta_{0}^{2} \alpha\left[k_{i}+\Gamma \beta_{0}^{2} p_{22}-\Gamma \beta_{0}^{2} \alpha\left(k_{p}-\Gamma \beta_{0}^{2} \alpha\right)\right]
\end{gathered}
$$

For $\Gamma \beta_{0}^{2} p_{22} \gg k_{i}$, which corresponds to fast adaptation and or large persistent excitation, then

$$
\begin{gathered}
\alpha=\left(\Gamma \beta_{0}^{2} p_{22}\right)^{-1} p_{12} \\
r=-p_{22}^{-1} p_{12}\left[k_{i}-p_{22}^{-1} p_{12}\left(k_{p}-p_{22}^{-1} p_{12}\right)\right]
\end{gathered}
$$

For the ideal tracking error response with $A_{p}=A$ and $B_{p}=B$, the characteristic equation is second-order with $a=0$ and $\Gamma=0$ in Eq. (50). For the system to have good damping characteristics, the closed-loop poles should be a complex-conjugate pair. This implies $k_{i} \geq \frac{k_{p}^{2}}{4}$ in order for $\operatorname{Re}\left[-\lambda_{\max }\left(A_{c}\right)\right]$ to be largest. Then

$$
p_{22}^{-1} p_{12}=k_{p}\left(1+k_{i}\right)^{-1} \leq k_{p}\left(1+\frac{k_{p}^{2}}{4}\right)^{-1}
$$

Thus $r$ is relatively small if $k_{p}$ is sufficiently large and therefore can be neglected. Then, the approximate roots of the characteristic equation (50) are

$$
\begin{gathered}
s=-a \\
s=-\Gamma \beta_{0}^{2} \alpha=-p_{22}^{-1} p_{12} \\
s=-\frac{\bar{k}_{p}}{2} \pm j\left(\bar{k}_{i}-\frac{\bar{k}_{p}^{2}}{4}\right)
\end{gathered}
$$

where $\bar{k}_{p}$ and $\bar{k}_{i}$ are the linearly approximate adaptive proportional and integral gains defined as

$$
\begin{gathered}
\bar{k}_{p}=k_{p}-p_{22}^{-1} p_{12} \\
\bar{k}_{i}=k_{i}+\Gamma \beta_{0}^{2} p_{22}-p_{22}^{-1} p_{12}\left(k_{p}-p_{22}^{-1} p_{12}\right)
\end{gathered}
$$

Equation (58) reveals that as $\Gamma \beta_{0}^{2}$ increases for fast adaptation and or large persistent excitation, the imaginary part of the complex-conjugate poles becomes large. Consequently, fast adaptation will result in high frequency oscillations in adaptive signals, a well-known fact in adaptive control [8]. 
This high frequency oscillation can result in excitation of unmodeled dynamics that may be present in the system and therefore can lead to a possibility of instability. The approximate bounded linear stability method is able to capture this behavior of nonlinear adaptive control in the linear analysis context. This method should be able to provide a method for assessing linear stability margins that can be used to adjust the adaptive gain.

\section{Metrics-Driven Adaptive control}

Metric-driven adaptive control is an approach that addresses stability and robustness of adaptive control in terms of quantifiable metrics. The goal of metrics-driven adaptive control is to achieve adaptation that satisfies a given set of metrics. Since adaptive control is nonlinear, the notion of metrics is not well established. Lacking of appropriate metrics for nonlinear adaptive control, the bounded linear stability analysis method can provide a framework for metricsdriven adaptive control whereby the nonlinear adaptive law is approximated by a linear equivalent system. Stability of the approximate LTI system can then be quantified in terms of gain and phase margins. These margins define how close to the verge of instability a control system is when subjected to disturbances. The adaptive gain can then be estimated from the bounded linear stability analysis method to meet specified margins and then used to drive the adaptation. Based on this approach, the system transfer function is obtained by taking the Laplace transform of the plant model as

$$
\begin{aligned}
s x=\left(A_{p}\right. & \left.-B_{p} \hat{B}_{p}^{*-1} \hat{A}_{p}^{*}\right) x+B \hat{B}_{p}^{*-1}\left(-b^{\top} A_{c}+\frac{\Gamma \beta_{0}^{2} b^{\top} P}{s}\right) e \\
& +B \hat{B}_{p}^{*-1}\left(s x_{m}-W^{* \top} \beta-\frac{-a z_{2}+\varepsilon_{1}+\varepsilon_{2}}{s}\right)
\end{aligned}
$$

The open-loop transfer function between $x$ and $\tilde{x}$ is

$$
\begin{aligned}
G(s)= & \left(s I-A_{p}+B_{p} \hat{B}_{p}^{*-1} \hat{A}_{p}^{*}\right)^{-1} \times \\
& \times B_{p} \hat{B}_{p}^{*-1}\left(K_{p}+\frac{K_{i}+\Gamma \beta_{0}^{2} P_{22}}{s}+\frac{\Gamma \beta_{0}^{2} P_{12}}{s^{2}}\right)
\end{aligned}
$$

The RLS indirect adaptive law results in convergence of $\hat{A}_{p}^{*} \rightarrow A_{p}$ and $\hat{B}_{p}^{*} \rightarrow B_{p}$ if $a \rightarrow 1$. Then, the transfer function becomes

$$
G^{*}(s) \approx \frac{K_{p} s^{2}+\left(K_{i}+\Gamma \beta_{0}^{2} P_{22}\right) s+\Gamma \beta_{0}^{2} P_{12}}{s^{3}}
$$

$G^{*}(s)$ can be broken into individual SISO transfer functions from which stability margins can be computed. Stability margins of $G(s)$ can be evaluated by structured singular values, or by one loop at a time and then the worstcase stability margins could be estimated using multi-loop stability margin definitions [9]. The stability margins are generally functions of $\Gamma \beta_{0}^{2}$. The persistent excitation $\beta_{0}^{2}$ can be computed from Eq. (27) within a given time window. Using this value, the adaptive gain $\Gamma$ can be calculated and used for adaptation for the next time window. This process is repeated until $\Gamma$ should reach a steady state value when the weights no longer vary.

\section{SIMULATION}

To illustrate the bounded linear stability analysis method, a simulation was performed for a damaged twin-engine generic aircraft with $25 \%$ of the left wing missing [2], as shown in Fig. 2. The hybrid adaptive control is implemented in a flight control to track a pitch doublet.

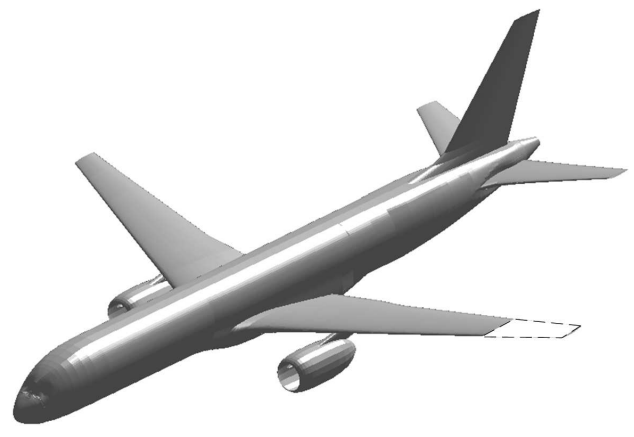

Fig. 2 - Damaged Generic Aircraft

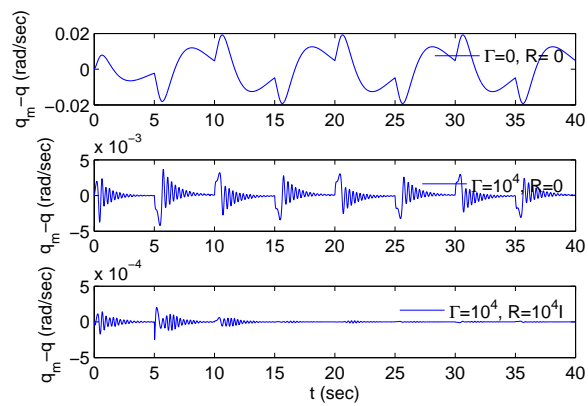

Fig. 3 - Pitch Rate Tracking Error

Figure 3 is a plot of the pitch rate tracking error. Without adaptation $(\Gamma=0, R=0)$, the tracking performance of the flight control is quite poor as the tracking error is large. With the direct MRAC alone $\left(\Gamma=10^{4}, R=0\right)$, the tracking error becomes smaller but high frequency contents also appear. This is consistent with the closed-loop pole analysis. With the hybrid adaptive control $\left(\Gamma=10^{4}, R=10^{4} I\right)$, the tracking error is significantly reduced along with the high frequency contents. Thus, the hybrid adaptive control appears to be more effective than the direct MRAC alone

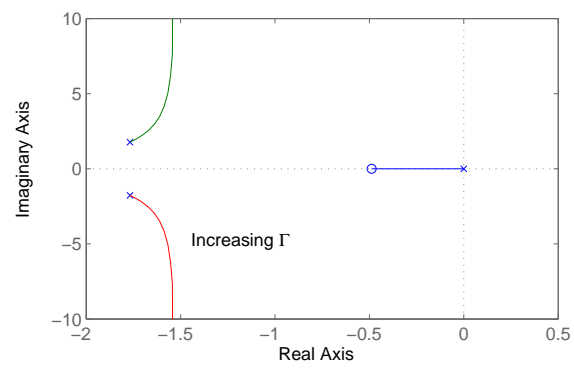

Fig. 4 - Root Locus for Pitch Loop

Figure 4 is the root locus plot of the characteristic equation for $a=1$ when $R_{0} \Phi_{0}^{2} \gg 1$. The root locus plot agrees well 
with the closed-loop pole analysis. The imaginary part of the complex-conjugate poles increases with increasing the adaptive gain $\Gamma$. This gives rise to high frequency oscillations in the adaptive signals when $\Gamma$ is large.

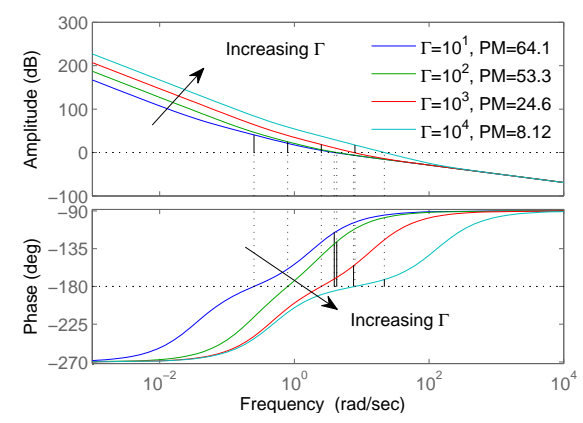

Fig. 5 - Bode Plot of $G^{*}(s)$ of Pitch Loop

Figure 5 is the Bode plot of the transfer functions $G^{*}(s)$ evaluated for the first 5 seconds. The Bode plot shows that as $\Gamma$ increases, the phase margin deteriorates. This is a typical behavior of a high-gain controller. Thus, while increasing $\Gamma$ leads to a better tracking performance, the relative stability of the system is compromised, as high frequency signals can excite unmodeled dynamics and lead to instability [7].

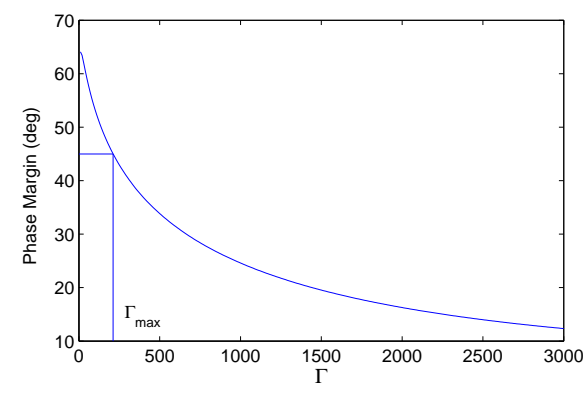

Fig. 6 - Phase margin

Figure 6 is a plot of the phase margin versus $\Gamma$. Increasing $\Gamma$ causes the phase margin to decrease. MIL-F-9490 specification for flight control systems typically requires a phase margin of $45^{\circ}$ and a gain margin of $6 \mathrm{~dB}$. The adaptive gain $\Gamma$ corresponding to this phase margin specification establishes an upper bound $\Gamma_{\max }$ for metrics-driven adaptive control.
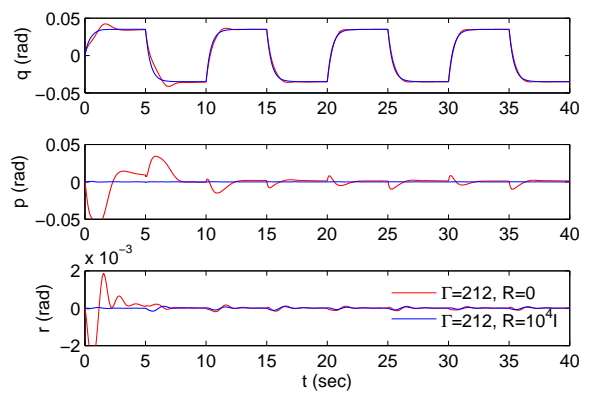

Fig. 7 - Metrics-Driven Hybrid Adaptive Control
Figure 7 is a plot of the pitch rate doublet tracking and roll and yaw rate responses to meet a phase margin of $45^{\circ}$ with an adaptive gain $\Gamma=\Gamma_{\max }$. The hybrid adaptive control ( $\Gamma=\Gamma_{\max }, R=10^{4} I$ ) clearly performs better than the direct MRAC alone $\left(\Gamma=\Gamma_{\max }, R=0\right)$, which suffers large initial transients, although high frequency contents no longer appear in the signals.

\section{CONCLUSIONS}

This paper has presented a bounded linear stability analysis method for analyzing approximate linear stability margins of a nonlinear hybrid adaptive control that blends both direct and recursive least-squares indirect adaptive laws. A piecewise, approximate linear equivalent system is formulated over a short, moving time windows within which the stability margins are analyzed. The analysis relates the convergence of the tracking error with the persistent excitation for the direct adaptive law, and the parameter convergence of the plant model with the persistent excitation for the indirect adaptive law. The closed-loop poles of the approximate linear equivalent system shows that increasing the adaptive gain results in high-frequency oscillations in the adaptive signals. A margin analysis shows that increasing the adaptive gain causes the phase margin to decrease. Thus, there exists an upper bound for an adaptive gain that satisfies a specified phase margin. This adaptive gain is used to limit the direct adaptation in the hybrid adaptive control to provide robustness. The simulation shows that the metrics-driven hybrid adaptive control has a better tracking performance than the direct adaptive control alone.

\section{REFERENCES}

[1] Totah, J., Krishnakumar, K., and Vikien, S., "Integrated Resilient Aircraft Control - Stability, Maneuverability, and Safe Landing in the Presence of Adverse Conditions", NASA Aeronautics Research Mission Directorate Aviation Safety Program, April 13, 2007.

[2] Nguyen, N., Krishnakumar, K., Kaneshige, J., and Nespeca, P., "Dynamics and Adaptive Control for Stability Recovery of Damaged Asymmetric Aircraft", AIAA Guidance, Navigation, and Control Conference, AIAA-2006-6049, 2006.

[3] Rysdyk, R.T. and Calise, A.J., "Fault Tolerant Flight Control via Adaptive Neural Network Augmentation", AIAA Guidance, Navigation, and Control Conference, AIAA-1998-4483, 1998.

[4] Johnson, E.N., Calise, A.J., El-Shirbiny, H.A., and Rysdyk, R.T., "Feedback Linearization with Neural Network Augmentation Applied to X-33 Attitude Control", AIAA Guidance, Navigation, and Control Conference, AIAA-2000-4157, 2000.

[5] Hovakimyan, N., Kim, N., Calise, A.J., Prasad, J.V.R., and Corban, E.J., "Adaptive Output Feedback for High-Bandwidth Control of an Unmanned Helicopter", AIAA Guidance, Navigation and Control Conference, AIAA-2001-4181, 2001.

[6] Eberhart, R.L. and Ward, D.G., "Indirect Adaptive Flight Control System Interactions", International Journal of Robust and Nonlinear Control, Vol. 9, pp. 1013-1031, 1999.

[7] Ioannu, P.A. and Sun, J. Robust Adaptive Control, Prentice-Hall, 1996

[8] Cao, C., Patel, V.V., Reddy, C.K., Hovakimyan, N., Lavretsky, E., and Wise, K., "Are Phase and Time-Delay Margin Always Adversely Affected by High Gains?", AIAA Guidance, Navigation, and Control Conference, AIAA-2006-6347, 2006.

[9] Anderson, M.R., Rabin, U.H., and Vincent, J.H., "Evaluation Methods for Complex Flight Control Systems", AIAA Guidance, Navigation, and Control Conference, AIAA-1989-3502, 1989. 